\title{
Assessment of NPP VIIRS Ocean Color Data Products: Hope and Risk
}

\author{
Kevin R. Turpie ${ }^{* a}$, Gerhard Meister ${ }^{\mathrm{b}}$, Gene Eplee ${ }^{\mathrm{a}}$, Robert A. Barnes ${ }^{\mathrm{a}}$ \\ Bryan Franz ${ }^{\mathrm{b}}$, Frederick S. Patt ${ }^{\mathrm{a}}$, Wayne D. Robinson ${ }^{\mathrm{a}}$, Charles R. McClain ${ }^{\mathrm{b}}$ \\ ${ }^{a}$ Science Applications International Corporation, Beltsville, Maryland 20705, USA \\ ${ }^{\mathrm{b}}$ NASA Goddard Space Flight Center, Greenbelt, Maryland 20771, USA
}

\begin{abstract}
For several years, the NASA/Goddard Space Flight Center (GSFC) NPP VIIRS Ocean Science Team (VOST) provided substantial scientific input to the NPP project regarding the use of Visible Infrared Imaging Radiometer Suite (VIIRS) to create science quality ocean color data products. This work has culminated into an assessment of the NPP project and the VIIRS instrument's capability to produce science quality Ocean Color data products. The VOST concluded that many characteristics were similar to earlier instruments, including SeaWiFS or MODIS Aqua. Though instrument performance and calibration risks do exist, it was concluded that programmatic and algorithm issues dominate concerns.
\end{abstract}

Keywords: NPP, VIIRS, Ocean Color, satellite remote sensing, climate data record.

\section{INTRODUCTION}

In 1994, three US Government organizations, namely the National Oceanic and Atmospheric Administration (NOAA), Department of Defense (DoD), and the National Aeronautics and Space Administration (NASA), created a joint program called the Integrated Project Office (IPO) to develop and manage the National Polar-orbiting Operational Environmental Satellite System (NPOESS). This program would put a suite of instruments into orbit every few years, maintaining multiple polar-orbiting environmental satellites for operational weather "now-casting" and forecasting, and for other oceanic and terrestrial applications. Each of these satellites will carry the Visible/Infrared Imager Radiometer Suite (VIIRS), which would provide 22 science bands at $750 \mathrm{~m}$ at nadir resolution, including seven ocean-color bands at visible and NIR wavelengths. VIIRS is similar to the MODerate resolution Imaging Spectroradiometer (MODIS) from NASA's Earth Observing System (EOS) missions, Aqua and Terra, both having two-dimensional focal planes, but it makes use of rotating telescope that is similar to the one used with Sea-viewing Wide Field-of-View Sensor (SeaWiFS). The first satellite to be launched is part of a risk reduction, data continuity mission call the NPOESS Preparatory Project (NPP) and represents the USA's only ocean-color capable mission for at least the next eight years, given a projected launch for the Pre- Aerosol, Cloud and ocean Ecology (PACE) instrument near the end of the decade.

A primary objective of NASA for the NPP mission is to extend the accurate and consistent time series of terrestrial, oceanic, and atmospheric observations currently produced from MODIS. This includes Ocean Color data, which are useful for understanding the role of the ocean biosphere in the Earth system at seasonal-to-decadal time scales ${ }^{1,2}$. NASA's Ocean Color record began with the Coastal Zone Color Scanner (CZCS), was resumed with SeaWiFS, and is being continued with MODIS Aqua. To determine whether Ocean Color data record continuity can be met with NPP VIIRS, NASA is evaluating and monitoring the mission. The study presented here was carried out by the NASA/Goddard Space Flight Center (GSFC) NPP VIIRS Ocean Science Team (VOST). The conclusions drawn from information available prior to launch are summarized in this paper ${ }^{3}$.

Because the quality of Ocean Color is highly dependent on the quality of the prelaunch characterization of the instrument, particular attention was given to understanding of sensor characteristics such as crosstalk, out-of-band (OOB) response, stray light, and polarization sensitivity. The evaluation, however, looked at other key factors in data product quality, including calibration techniques and issues, algorithms, and processing infrastructure and resource

* kevin.r.turpie@nasa.gov; phone 1301286 9996; fax 1301286 0268; oceancolor.gsfc.nasa.gov 
application.

It should be noted, however, that no prelaunch assessment can guarantee that VIIRS products will achieve a level of accuracy and consistency presently derived from SeaWiFS and MODIS data, which would be required for NPP Ocean Color data products to meet NASA objectives for climate and Earth System Science research, as well as Earth Observing System (EOS) climate data continuity. For climate research using satellite Ocean Color climate data records (CDR), an on-orbit calibration stability of $0.1 \%$ over the mission lifetime is required, and that achievement can only be assessed retrospectively. Furthermore, long-term instrument performance is only one requirement towards meeting NASA science and climate objectives. To continue Earth System Science and climate research at NASA, minimum scientific requirements also include, but are not limited to:

1. Sensor data quality comparable to heritage EOS-era instruments,

2. State of the art data product algorithms consistent with those currently employed by EOS instruments,

3. Inter-agency investment in post-launch vicarious and on-board calibration and validation (Cal/Val) activities (e.g., field sites), including approving spacecraft lunar maneuvers as executed by heritage missions, and

4. The capability for mission-level data reprocessing.

This paper describes concerns regarding these requirements; concerns which put EOS data continuity objectives at risk. Although actual flight data will be the ultimate proof, the authors conclude from prelaunch testing and instrument characterization that NPP VIIRS data could be of a quality that is within reach of standards set by heritage missions (e.g., MODIS or SeaWiFS). Instead, the key risks to sustaining the current climate record relate to algorithms and algorithm support, calibration and validation activity support, and support for a mission-level reprocessing capability. However, resources and infrastructure can still be brought to bear to mitigate these major risks. Therefore, the researchers interested in studying connections between climate and the ocean biosphere have some hope of getting the level of quality that they need, provided that such mitigations are done.

\subsection{Processing System}

\section{EVALUTION}

A recommended minimum requirement for a consistent Ocean Color CDR, or any $C D R$, is mission-level data reprocessing. As was the case for MODIS Aqua, VIIRS will have a sun-glinted swath and a single calibration buoy. Because of those constraints, it took MODIS Aqua about three years to accumulate enough data to converge on stable vicarious calibration corrections ${ }^{4}$. Once these corrections were obtained, the previous three years of data needed to be reprocessed to provide a consistent data record. Similarly, any changes to instrument behavior on-orbit, or deviation from prelaunch understanding of known anomalies, would require either new corrections or modification to existing ones. For heritage instruments, this process can require a lengthy time series, as was the experience with MODIS, on the EOS satellites Aqua ${ }^{5}$ and Terra, ${ }^{6}$ in determining the polarization response on-orbit. To produce a consistent data record, any new or revised correction will need to be applied retrospectively. An Ocean Color CDR cannot be produced without mission-level reprocessing.

\subsection{Algorithms}

Data product consistency is a fundamental CDR requirement ${ }^{7}$. Currently, the NPP operational algorithms are older and less rigorous than those currently used by NASA (e.g., MODIS, SeaWiFS) to generate its Ocean Color CDR products. For instance, although based fundamentally on the same theory of Gordon and Wang ${ }^{8,9}$, the operational NPP algorithm for atmospheric correction over the ocean is several years behind the current NASA algorithm ${ }^{10}$. Furthermore, the current NPP algorithm for chlorophyll $a$ concentration ${ }^{11}$ is not only completely inconsistent with the one used by NASA $^{12}$ to produce its climate data record, it has been demonstrated using validation data that it simply will not perform as well, especially in coastal regions ${ }^{13}$. Thus the current operational NPP algorithm for chlorophyll $a$ also cannot be expected to produce data of the quality that are commensurate or even compatible with the current climate data record. A more current NASA algorithm is expected to be inserted sometime after launch, but that would imply a considerable gap 
in the climate record, especially without reprocessing support. Furthermore, the performance of the chlorophyll algorithm will be limited by the quality of the atmospheric correction. Therefore, the operational data processing is expected to yield results that are neither consistent with nor of the same level of quality as those found in current NASA production software. Given the current operational NPP/NPOESS Ocean Color Environmental Data Record (EDR) algorithms, it is clear that the Ocean Color CDRs cannot be continued with the operational NPP EDRs.

Table 1 - Listed are key developments to the Ocean Color processing. The R column indicates the status of the research algorithm or methodology developed at NASA/GSFC. The O column refers to the status of the NPP operational algorithm. (X) indicates that the capability is fully tested and vetted. (-) indicates that the capability is still under development, exists but has not benefited from developments at NASA/GSFC, or has not been consistently developed to the NASA standard. A blank entry indicates that the capability is missing or completely deficient.

\begin{tabular}{|c|c|c|c|}
\hline Development & Description & $\mathbf{R}$ & $\mathbf{O}$ \\
\hline NIR Correction & $\begin{array}{l}\text { Important for turbid and productive waters (e.g., coastal waters). } \\
\text { This is an iterative process that may impact latency requirements } \\
\text { for the operational stream. }\end{array}$ & $\mathrm{X}$ & \\
\hline Aerosol Models & $\begin{array}{l}\text { GSFC improved aerosol correction using relative humidity } \\
\text { based aerosol selection scheme of } 80 \text { AeroNET-band aerosol } \\
\text { models. These improvements are not in the operational } \\
\text { algorithms. }\end{array}$ & $\mathrm{X}$ & - \\
\hline $\begin{array}{l}\text { Polarization } \\
\text { Correction }\end{array}$ & $\begin{array}{l}\text { This is crucial to account for variation in the instrument } \\
\text { response to Top-of-Atmosphere (TOA) polarization. Both } \\
\text { operational and research streams have this correction. }\end{array}$ & $\mathrm{X}$ & $\mathrm{X}$ \\
\hline $\begin{array}{l}\text { Trace Gas } \\
\text { Absorption }\end{array}$ & $\begin{array}{l}\mathrm{NO}_{2} \text { Corrections; updated source spectra for } \mathrm{O}_{3} \text { cross-section; } \\
\text { other gaseous absorption included for SeaWiFS. Operational } \\
\text { version is not tested; proper and consistent use of ancillary data } \\
\text { is not clear. }\end{array}$ & $\mathrm{X}$ & - \\
\hline $\begin{array}{l}\text { Sun Glint } \\
\text { Correction }\end{array}$ & $\begin{array}{l}\text { Crucial to improve data coverage; Wang-Bailey algorithm } \\
\text { provided to contractor in } 2005 \text { for operational algorithm (testing } \\
\text { status is unknown). }\end{array}$ & $\mathrm{X}$ & - \\
\hline $\begin{array}{l}\text { Updated Rayleigh } \\
\text { Model }\end{array}$ & $\begin{array}{l}\text { Updated tables, pressure corrections, and source spectra. VIIRS } \\
\text { version may require additional corrections (e.g., detector-to- } \\
\text { detector or crosstalk specific relative spectral response) and both } \\
\text { operational and research teams are looking at this. }\end{array}$ & - & - \\
\hline $\begin{array}{l}\text { Revised Whitecap } \\
\text { Algorithm }\end{array}$ & $\begin{array}{l}\text { This calculation of the reflectance from whitecaps was improved } \\
\text { over the version still found in the operational version. }\end{array}$ & $\mathrm{X}$ & - \\
\hline $\begin{array}{l}\text { VIIRS Crosstalk } \\
\text { Correction }\end{array}$ & $\begin{array}{l}\text { A method was developed by the operational team, but has not } \\
\text { been tested and could have significant limitations. However, the } \\
\text { method is simple to implement in the research-processing } \\
\text { stream. }\end{array}$ & - & - \\
\hline OOB Correction & $\begin{array}{l}\text { Based on best relative spectral response (RSR) curves available, } \\
\text { band-pass correction and Rayleigh tables are adjusted for out-of- } \\
\text { band (OOB) light leaks. }\end{array}$ & - & - \\
\hline $\begin{array}{l}\text { Band-Pass } \\
\text { Corrections }\end{array}$ & $\begin{array}{l}\text { Necessary to account for differences between ground and space } \\
\text { sensor band-passes. Lw band-pass corrections based on latest } \\
\text { Morel bio-optical model. }\end{array}$ & $\mathrm{X}$ & \\
\hline BRDF Corrections & $\begin{array}{l}\text { Necessary correction to account for geometric effects of in-water } \\
\text { reflectance. Changes included upwelling/downwelling } \\
\text { reflection/refraction and f/Q. }\end{array}$ & $\mathrm{X}$ & \\
\hline $\begin{array}{l}\text { New K490 } \\
\text { Algorithm }\end{array}$ & $\begin{array}{l}\text { The extinction coefficient at 490nm was revised and tuned with } \\
\text { the NOMAD V2 dataset. }\end{array}$ & $\mathrm{X}$ & \\
\hline $\begin{array}{l}\text { Tuned Chl } a \\
\text { Algorithm }\end{array}$ & $\begin{array}{l}\text { Algorithm is tuned using NOMAD V2 dataset developed by } \\
\text { GSFC. Operational team has added research algorithm, but has } \\
\text { not tuned it. }\end{array}$ & $\mathrm{X}$ & - \\
\hline $\begin{array}{l}\text { Produce POC and } \\
\text { PIC }\end{array}$ & $\begin{array}{l}\text { Particular organic carbon and particular inorganic carbon are } \\
\text { now standard products in the GSFC research-processing stream. }\end{array}$ & $\mathrm{X}$ & \\
\hline $\begin{array}{l}\text { Report Rrs instead } \\
\text { of } n L w\end{array}$ & $\begin{array}{l}\text { Remote sensing reflectance is now reported as a standard } \\
\text { product instead of nLw to support community needs. }\end{array}$ & $\mathrm{X}$ & \\
\hline
\end{tabular}




\begin{tabular}{|l|l|l|l|}
\hline $\begin{array}{l}\text { Angstrom Exponent } \\
\text { and AOT }\end{array}$ & $\begin{array}{l}\text { Angstrom exponent and aerosol optical thickness (AOT) are } \\
\text { now standard products of the GSFC research-processing stream. }\end{array}$ & $\mathrm{X}$ & \\
\hline Produce PAR & $\begin{array}{l}\text { Photosynthetically available radiation (PAR) is now a standard } \\
\text { product of the GSFC research-processing stream. }\end{array}$ & $\mathrm{X}$ & \\
\hline Research Products & $\begin{array}{l}\text { Numerous other data products, such as semi-analytic models of } \\
\text { chlorophyll and inherent optical properties (IOP), are available } \\
\text { through GSFC software. Operational processing stream } \\
\text { provides Carder Chl a }{ }^{11} \text { and IOP parameters as standard EDR } \\
\text { products. }\end{array}$ & $\mathrm{X}$ & - \\
\hline $\begin{array}{l}\text { Vicarious } \\
\text { Calibration } \\
\text { Methodology }\end{array}$ & $\begin{array}{l}\text { Considerable research was done to develop the techniques to use } \\
\text { calibration buoy measurements and atmospheric correction } \\
\text { models to determine static gain biases. It is not clear whether } \\
\text { this is being applied properly for the operational product. }\end{array}$ & $\mathrm{X}$ & - \\
\hline
\end{tabular}

Table 1 compares capabilities that are found in the research-processing stream at NASA/GSFC to the NPP operational processing stream. Many corrections are missing from the NPP operational processing stream, such as the NIR correction. It is suspected that this correction, which is iterative, might be viewed as prohibitive for the operational use as it can be computationally expensive. In addition, a correction for gaseous absorption was presumably added recently to the NPP operational processing stream, though it is not clear whether it will work as effectively as the heritage correction, which has been validated with flight data. The six items preceding "Research Products" include data products that are now standard for research processing stream at NASA/GSFC, which are not included in the operational Ocean Color EDR product suite. For both the NASA research algorithms and NPP operational algorithms, the methods for addressing the idiosyncratic spectral and crosstalk characteristics of NPP VIIRS are based on the techniques used for heritage missions. But, in either case, these remain largely untested and are marked as still being under development.

The OC3 chlorophyll algorithm ${ }^{12}$ has been inserted into the NPP operational stream, but this has not yet been tuned to the VIIRS band-pass and there are currently no plans to do so using the second and most recent version of the standard NASA dataset known as the NASA bio-Optical Marine Algorithm Dataset (NOMAD V2). Moreover, the OC3 algorithm has only been placed in the operational processing for further study and there is no current plan to generate chlorophyll concentrations with this algorithm on a regular basis. Therefore, there is no indication that the operational algorithm will ever be consistent with the current climate data record for chlorophyll $a$ concentration.

\subsection{Instrument Characterization}

CDR production requires the highest quality data with well quantified and understood uncertainties. ${ }^{2}$ Many VIIRS instrument characteristics, or their analyses by other teams, were reviewed and it was determined that no corresponding adverse affects were expected for the data quality. However, a number of issues were identified that were viewed as a risk to the applicability of VIIRS to climate research. After analysis of the characterization data, however, most radiometric characteristics were found to have little to no significant impact to data quality.

In addition to variation in detector gains, effects like crosstalk or instrument spectral response smile are expected to lead to striping in the Ocean Color EDR. Some corrections may reduce this problem, such as a point-to-point crosstalk correction or an along-track correction for instrument smile, but some residual striping will be inevitable and could be difficult to remove. However, striping is not expected to be a limiting factor for most applications of the Ocean Color EDR. NFR, crosstalk, and other sources of extraneous signal are expected to affect data coverage around cloud edges and coastlines. Although there is no clear evidence that this will be any worse than with heritage missions, the impact of cloud-contaminated pixels will need to be carefully evaluated on-orbit.

Special attention was given for the issue of a low signal-to-noise ratio (SNR) and the existence of radiometric response anomalies for radiance levels near the gain switch point for dual gain bands. VIIRS samples are roughly $250 \mathrm{~m}$ in scan direction at nadir. These are aggregated three-to-one over the larger portion of the middle swath. Depending on band, this boosts the SNR to be somewhere between the SNR achieved by the SeaWiFS and MODIS instruments. Further out on the swath, the aggregation drops to two-to-one and no aggregation is done toward the swath edges and the SNR subsequently drops. When the expected noise is averages over all these different aggregation zone, the overall SNR in 
the data tends to be closer to SeaWiFS.

The analyses for several spatial performance areas were also reviewed, including band-to-band registration, near-field response, and the spatial effects of crosstalk. In general, none of the spatial effects are expected to cause significant issues. However, further analysis of the spatial characteristics of crosstalk may be warranted. Furthermore, although prelaunch data appear to vindicate VIIRS regarding any issues of stray light, some project engineers have expressed concern with the tests that produced those data. Therefore, the authors conclude from those data that stray light performance should be comparable to MODIS, but reserve some doubt until flight data can be examined postlaunch.

The area that may present the greatest challenge for VIIRS as an Ocean Color instrument is its ability to get the same measurement for two identical radiance values in a given band when the two target spectra are different at other wavelengths. Early measurements showed that the VIIRS Flight Unit (FU1) Integrated Filter Assembly (IFA) has spectral performance issues that could affect the ability to consistently calibrate the instrument to a level commensurate with heritage performance. The key issue was the presence of optical crosstalk between bands on the Visible/NearInfrared focal plane. However, the level of impact for IFA issues is less than was indicated by the Engineering Design Unit (EDU) or earlier FU1 tests. For FU1, a decision was made to flip the filter array to trade crosstalk effects with OOB light leaks, which may be easier to correct on-orbit, which is facilitated by the rigorous characterization the IFA. Effort continues toward estimation of IFA characterization uncertainties remains incomplete. For instance, polarization sensitivity of spectral response and aspects of crosstalk are not included in the spectral response curves, which compound uncertainty for an on-orbit correction. However, there is hoped that these effects will be sufficiently small as to not undermine corrections for IFA behavior.

\subsection{Calibration}

\subsubsection{AOI Dependent Degradation}

MODIS views the Solar Diffuser (SD), a reference panel compose of Spectralon ${ }^{\circledR}$, at an angle of incidence (AOI) on the primary scan mirror equivalent to the start of the second half of the earth view scan, and it views the moon at an AOI equivalent to the very beginning of the earth view scan. VIIRS views both the SD and the moon at an AOI on its halfangle mirror slightly outside the AOIs of the earth view. The AOI is identical for the lunar and SD view. Therefore, it will not be possible to use the lunar and SD measurements to correct for response versus scan angle variations. This has been critical for the MODIS visible bands (e.g. a 20\% effect on the MODIS Aqua 412nm band). The VIIRS telescope design is similar to the SeaWiFS design, where the HAM is well protected and has not shown a significant AOI dependent degradation. Therefore, the assumption is that the VIIRS HAM will not show any AOI dependent degradation either. It is not clear how much confidence can be put into this assumption, but the assumption is not unreasonable. Conversely, if such degradation occurs, then there is no on-board calibration mechanism to correct for such effects. It may be possible to correct such effects, e.g., with the methods developed at GSFC ${ }^{6}$, but it is not clear what truth data will be available for VIIRS (neither SeaWiFS nor MODIS Aqua may be available at the end of the VIIRS mission), and such corrections are very difficult to implement for an operational mission (due to the need to predict the corrections into the future using data acquired in the past).

\subsubsection{Solar Diffuser Reflectance Degradation}

The MODIS design protects the SD from solar illumination with a door that is only opened for the actual calibration events (approximately every two weeks). VIIRS calibration events occur every orbit, so there is no door to protect the SD. The NPP VIIRS SD is also oriented toward the direction of flight, which may further accelerate degradation. Both this issues suggest that the reflectance of the SD will degrade much faster than on MODIS. The Solar Diffuser Stability Monitor (SDSM) looks at the SD at a different angle than the VIIRS telescope. As long as the degradation of the reflectance of the SD is not angularly dependent, this is not a problem. If the change of reflectance measured by the SDSM is small, it is reasonable to assume that the effects with Bidirectional Reflectance Distribution Function (BRDF) are small as well. However, if the degradation measured by the SDSM is large, it is likely that the SD reflectance for the VIIRS telescope view angle is significantly different. These effects could be responsible for the corrections that need to be applied to the MODIS Terra SD measurements ${ }^{6}$. In that case, the MODIS Terra SD door became stuck open due to a mechanism malfunction in May 2003, leading to a large increase in the rate of degradation of the SD reflectance; both 
the SD reflectance degradation and the required corrections are largest at 412nm.

\subsubsection{Solar Diffuser Screen Characterization}

Unlike the MODIS design, VIIRS has a Solar Diffuser Screen (SDS) permanently in the light path. The problem with this approach is that the vignetting function of the SDS cannot be determined from on-orbit measurements (as it was done for MODIS by simply taking measurements with and without the SDS). The VIIRS vignetting function was measured prelaunch, but it remains to be seen whether these measurements are of sufficient quality. This concern stems from the fact that it is very challenging to accurately simulate solar-like illumination in the laboratory; attempts to illuminate the SD with solar-like illumination using NIST laser sources during the spacecraft level testing at Ball Aerospace in Spring 2010 were at best moderately successful.

\subsubsection{Lunar Calibration}

Lunar calibration is necessary to detrend changes in the instrument response over the course of the mission ${ }^{15,16,17}$. The on-orbit solar diffuser and lunar observations by VIIRS will be made at the same AOI on the HAM. The lunar calibration and solar diffuser time series will provide complementary data sets for monitoring the instrument's on-orbit radiometric performance. The overall on-orbit calibration of the instrument will require the development of analysis techniques to combine these two data sets, as was done for SeaWiFS early in its mission ${ }^{18}$. While the VIIRS calibration team is proceeding in the development of routines to analyze the VIIRS solar diffuser data, a similar development effort is required to extend the USGS RObotic Lunar Observatory (ROLO) photometric model of the Moon to work with VIIRS lunar data. A collaborative effort is underway between USGS and NASA to plan and implement analyses needed to support lunar calibration analysis. A non-exhaustive list of on-going and potential activities include the incorporation of the VIIRS RSR curves into the ROLO model to adapt the model output to the VIIRS bands and the development of an over-sampling correction scheme for the VIIRS lunar images. GSFC has also developed mission-planning software required to predict the lunar calibration opportunities needs to be implemented. The primary concern at this time is the incorporation of the results of these various analyses into the calibration processing of the VIIRS radiometric data products. Furthermore, decisions are still pending regarding whether NOAA will support the required roll maneuvers to acquire adequate data for tracking instrument radiometric response changes.

\subsubsection{Vicarious Calibration}

Vicarious calibration removes static biases in the radiometry, after being de-trended via lunar and solar calibration. This includes biases in both the instrument calibration and the atmospheric correction algorithm. The vicarious calibration process depends on matching up best quality satellite TOA radiance measurements with radiances derived from surface data that was taken at one or more calibration buoys and then subsequently converted into TOA radiance (using atmospheric radiance calculated by the radiative transfer algorithms used in the Ocean Color atmospheric correction).

Convergence of the vicarious calibration gain corrections to stable quantities is a function of overall data quality, and the number of data match-ups acquired. The number of usable data match-ups acquired for a given match-up protocol is a function of time and number of independent calibration sites in operation. Anomalies in the instrument performance for VIIRS that change from one match-up data pair to another, despite detrending with lunar calibration, will increase the number of data match-ups required to achieve convergence. Furthermore, biases that change as one moves away from the calibration site(s) (e.g., crosstalk) could decrease the effectiveness of the gain corrections in global applications.

With a single calibration site and NASA/GSFC vicarious calibration protocols, no heritage mission has achieved optimal stability for vicarious gain corrections for a given band in less than two years, and for MODIS Aqua some bands took as many as three years ${ }^{4}$. It is expected that the number of data match-ups for NPP VIIRS, like MODIS Aqua, will have less coverage of the ocean then SeaWiFS, because it is also a nadir pointing instrument that is subject to a substantially large sun glint pattern. Therefore, if a single calibration site is used, as it was for heritage missions and as described in current $\mathrm{Cal} / \mathrm{Val}$ plans, the rate of convergence under NASA/GSFC protocols is not expected to be any faster.

Furthermore, there are instrument performance issues that could impact the vicarious calibration convergence rate. VIIRS SNR levels are comparable to SeaWiFS and, as experienced with heritage instrument, instrument noise could also 
affect the convergence rate for vicarious gain corrections. To address this, special aggregation protocols may need to be developed to improve the convergence rate, if possible. Likewise, spectrally driven anomalies for NPP VIIRS (e.g., crosstalk) could affect the global effectiveness of gain corrections, depending on how well these effects can be removed. This would require further study to better quantify.

In addition, even a slight increase in the expected amount of data over the ocean that are significantly degraded or excluded around bright clouds can greatly reduce the amount of data available to perform a vicarious calibration. This relationship was studied by looking at the number of vicarious calibration data pairs for MODIS Aqua were acquired as pixels of an increasing distance from clouds were masked (see Figure 1). For VIIRS, this will also require an active investigation of the how much data is mask for stray light contamination versus possible correction strategies for crosstalk or NFR effects for the VIIRS data. Part of the trade analysis involves the number of match-up data required to perform a preliminary vicarious calibration and the further number of match-up data required to refine that calibration to achieve climate data records.

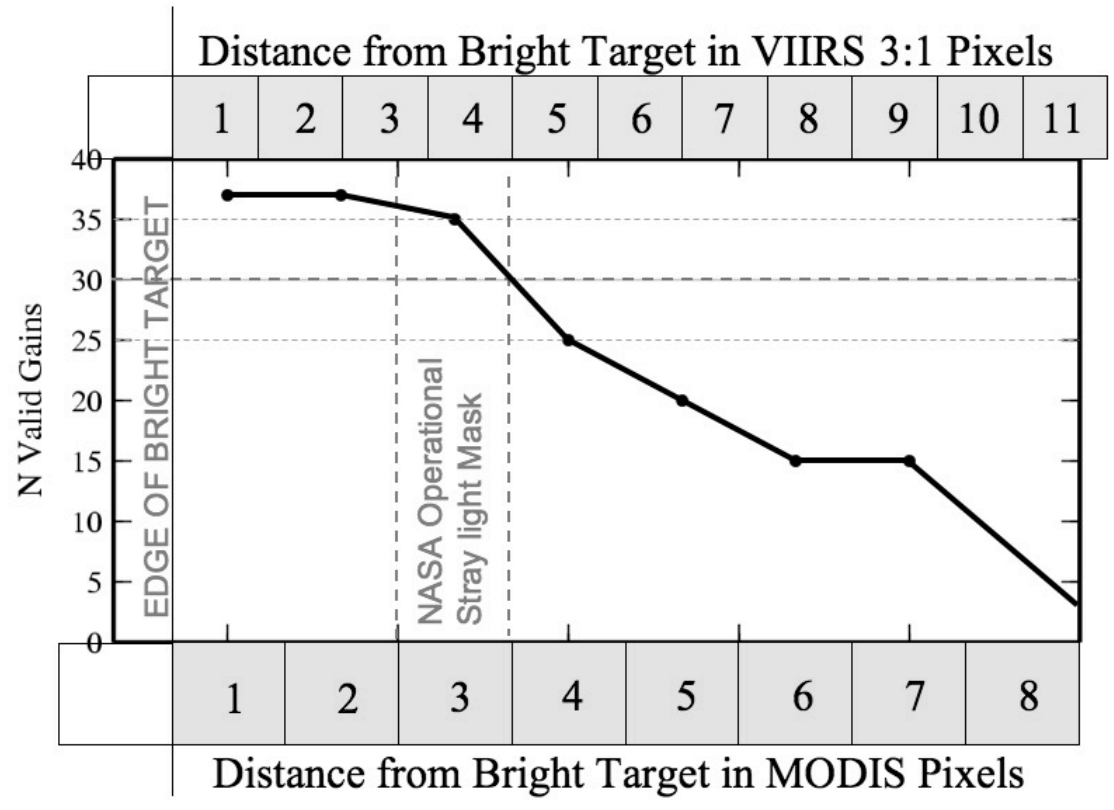

Figure 1. The broken line indicates the number of valid match-up pairs of satellite and calibration site data that were acquired for MODIS Aqua when the number of pixels (given by the number boxes along the bottom of the plot) were masked from cloud edges. The numbered boxes across the top give the equivalent in VIIRS 3:1 aggregation pixels. The dashed vertical lines indicate a range of mask sizes comparable to the $5 \times 7$ cloud pixel mask applied routinely by NASA/GSFC. The dashed horizontal lines indicate the number of match-up data pairs needed to achieve convergence of the gain corrections to stable quantities. The amount of available satellite data drops rapidly beyond seven MODIS pixels.

In general, it is recommended that further study be done to develop protocols for how data is selected for either vicarious calibration or validation. The NASA/GSFC protocols have the considerable experience of heritage mission, which would make them an ideal starting point for VIIRS. However, as mentioned, a number of VIIRS specific issues should be considered.

\subsection{Validation Risks}

The foremost risk to adequate validation of VIIRS Ocean Color data products is the support of a viable validation program. At the time of writing, approximately three months prior to launch, funding and continued support for the deployment of in-water assets (buoys, cruises, towers, etc.) and the analysis of the data acquired, appears precarious. Product validation is more than a metric to determine mission success. Validation provides the data with scientific context and meaning. 
Furthermore, the project defines conditions, or exclusion criteria, under which data product quality assessment is not required. This would imply that validation efforts would not be needed for data acquired by VIIRS under said conditions, including the collection of in situ data for certain regions. At the time that this paper is being written, the VIIRS data exclusion criteria for quality data are currently being re-developed. However, much of the previous criteria pertained to coastal conditions, including excluding waters with depths shallower than 50 meters (currently, being changed to $30 \mathrm{~m}$ ). The primary concern here is that much of the validation assets are in coastal regions. These are less expensive to deploy than deep-water assets and validation assets are sparse. Not collecting in situ data in coastal regions could significantly reduce the amount of usable data in the statistical sample, thus making successful validation of data performance increasingly less likely. Furthermore, current and future Ocean Color missions should strive to study and improve in-water retrievals for coastal regions as they are vastly important ecologically and economically and play an important role in human health and habitat.

\section{CONCLUSIONS}

The NPP VIIRS operational Ocean Color EDR data product, as currently supported, does not have sufficient quality to achieve NASA objectives for Earth System Science research, including EOS CDR data continuity. The primary reasons are the use of outdated algorithms that are not consistent with the current CDR and a lack of support for mission-level reprocessing. As with previous missions, there are also potential risks associated with instrument performance, characterization, and calibration. It is suggested that NASA objectives might be met with a separate research processing stream that supports NASA selected algorithms and mission-level reprocessing, provided other risks regarding instrument performance or calibration do not present problems on-orbit. For example, a full research processing capability is supported at NASA/GSFC to facilitate production of heritage data products and the evaluation of operational NPP EDR products.

The authors give considerable attention to VIIRS instrument performance because achieving high quality Ocean Color measurements was known to be a significant challenge in the development and characterization of heritage instruments. ${ }^{2}$ Detector-to-detector differences for radiometric and spectral response are expected to produce significant striping, a characteristic endemic to the instrument design type. NFR, crosstalk, and other sources of extraneous signal are expected to affect data coverage around cloud edges and coastlines. Although there is no clear evidence that this will be any worse than with heritage missions, the impact of cloud-contaminated pixels will need to be carefully evaluated onorbit. It was also noted that the instrument SNR is adequate for most Ocean Color applications with two to one or three to one sample aggregation, which is performed over much of the swath. Toward the edges of the swath, where no aggregation is done, the SNR is lower than heritage for some bands. Averaging noise over the entire swath, including all aggregation, the SNR is comparable to SeaWiFS. For NPP VIIRS, the margins above instrument specifications for SNR are very generous (i.e., $50-100 \%$ above specification thresholds). However, unless the specifications are tightened for SNR, margin could be lost for future VIIRS instruments. If an instrument had SNR at any lower level, then it insufficient for most NASA Ocean Color research objectives.

As with heritage instruments, many instrument performance issues were evaluated prelaunch for VIIRS. Just as with the heritage instruments, NPP VIIRS characterization test limitations and the potential for unforeseen changes in performance on-orbit limit the assessments and recommendations presented here. Any prelaunch assessment of VIIRS instrument performance does not guarantee that VIIRS data products will achieve a level of accuracy and consistency presently achieved with SeaWiFS and MODIS data. Therefore, the sensor should be carefully evaluated and monitored on-orbit for changes in performance that would impact Ocean Color measurements. It is also recommended that this be carried out collaboratively between an Ocean Color EDR and SDR calibration teams. Further work is suggested for: the evaluation of crosstalk and OOB light leak correction strategies, including the role of vicarious calibration; the stratification of the residual effects of crosstalk for conditions of interest; and a closer look at the spatial effects of crosstalk in coastal regions and bright algal blooms.

As described in Section 2.4, a number of concerns also exist with the on-orbit calibration and validation. Prelaunch system-level testing of the solar diffuser calibration system is not yet conclusive, so like MODIS, the function of NPP VIIRS calibration system continues to carry a prelaunch risk. The lack of an approved plan for calibration maneuvers throughout the operational mission, including roll maneuvers for lunar calibration and yaw maneuvers to characterize the calibration system, is a programmatic risk to NASA science objectives. Other calibration system issues that are described 
in Section 2.4 will need to be monitored on-orbit, where possible. The issues enumerated in Section 2.4 will need to be monitored during the Intensive Cal/Val (ICV) period of the mission, as feasible, by an Ocean Color EDR team.

\section{REFERENCES}

[1] Esaias, W.E., Abbott, M.R., Barton, I., Brown, O.B., Campbell, J.W., Carder, K.L., Clark, D.K., Evans, R.H., Hoge, F.E., Gordon, H.R., Balch, W.M., Letelier, R. Minnett, P.J., "An overview of MODIS capabilities for ocean science observations,” IEEE Transactions on Geoscience and Remote Sensing 36(4), 1250-1265 (1998).

[2] McClain, C.R, “A decade of satellite ocean color observations,” Annual Review of Marine Science 1, 19-42 (2009).

[3] Turpie, K.R., Meister, G., Eplee, R.E., Barnes, R.A., Patt, F.S., Robinson, W.D., McClain, C.R., "Ocean color data product assessment report for NPP VIIRS,” NASA/GSFC Greenbelt,MD, 53pp (2010).

[4] Franz, B.A., Bailey, S.W., Werdell, P.J., McClain, C.R., "Sensor-independent approach to the vicarious calibration of satellite ocean color radiometry,” Applied Optics 46(22). pp 5058-5082 (2007).

[5] Meister, G., Kwiatkowska, E., Franz, B.A., Patt, F.S., Feldman, G.C., McClain, C.R., "The MODIS ocean color polarization correction,” Applied Optics 44(26), pp 5524-5535 (2005).

[6] Kwiatkowska, E. J., Franz, B.A., Meister, G., McClain, C.R., Xiong, X., "Cross-calibration of ocean color bands from Moderate Resolution Imaging Spectroradiometer on Terra platform,” Applied Optics 47(36), pp 6796-6810 (2008).

[7] NRC (National Research Council), [Climate Data Records from Environmental Satellites]. Washington, D.C., National Academy Press (2004).

[8] Gordon, H.R., and Wang, M., "Retrieval of water-leaving radiance and aerosol optical thickness over the oceans with SeaWiFS: A preliminary algorithm,” Applied. Optics, 33, pp 443- 452 (1994).

[9] Northrop Grumman Corporation, [VIIRS Ocean Color/Chlorophyll Algorithm Theoretical Basis Document], Northrup Grumman, DOC D43763, Rev A, 52 pp (2010).

[10] Ahmad, Z., B.A. Franz, C.R. McClain, E.J. Kwaitkowska, J. eredell, E.P,. Shettle, and B.N. Holben, "New aerosol models for the retrieval of oaersol optical thickness and normalized water-leaving radiances from the SeaWiFS and MODIS sensors over coastal regions and open oceans," Appl. Opt. 49, 5545-5560 (2010).

[11] Carder, K.L., Chen, F.R., Lee, Z.P., Hawes, S.K., "Semianalytic Moderate- Resolution Imaging Spectrometer algorithms for chlorophyll $a$ and absorption with bio- optical domains based on nitrate-depletion temperatures,” Journal of Geophysical Research 104(C3), pp 5403-5421 (1999).

[12] O’Reilly, J.E., Maritorena, S., Mitchell, B.G., Siegel, D.A., Carder, K.L., Garver, S.A., Kahru, M., McClain, C.R., “Ocean color algorithms for SeaWiFS,” Journal of Geophysical Research 103(C11), pp 24937-24953 (1998).

[13] Signorini, S.R., McClain, C.R., Mannino, A., Bailey, S., [Report on Ocean Color and Carbon Study for the South Atlantic Bight and Chesapeake Bay Regions], NASA/TM-2005-212787, NASA Goddard Space Flight Center, Greenbelt, Maryland, 45 pp. (2005).

[14] Wang, M., Bailey, S.W., "Correction of sun glint contamination on the SeaWiFS ocean and atmosphere products," Applied Optics 40(27), pp 4790-4798 (2001).

[15] Sun, J., Eplee, R.E.Jr., Xiong, X., Stone, T., Meister, G., McClain, C.R., "MODIS and SeaWiFS on-orbit lunar calibration.” in Earth Observing Systems XIII, Proc. SPIE 7081, 70810Y (2008).

[16] Eplee, R.E., Jr., J.-Q. Sun, G. Meister, F.S. Patt, X. Xiong, and C.R. McClain, "Cross calibration of SeaWiFS and MODIS using on-orbit observations of th Moon," Applied Optics 50, 120-133 (2011). and R.E. Eplee, Jr., G. Meister, [17] F.S. Patt, Franz, B.A., and C.R. McClain, "Uncertainty assessment of the SeaWiFS on-orbit calibration," in Earth Observing Systems XVI, J.J. Butler, X. Xiong, and X. Gu, eds., Proc. SPIE 8153, 815310 (2011).

[18] Eplee, R.E.Jr., Meister, G., Patt, F.S., Bailey, S.W., McClain, C.R., “The on-orbit calibration of SeaWiFS,” (being prepared for Applied Optics) (2012). 